\title{
HabEx polarization ray trace and aberration analysis
}

Jeffrey Davis, Meredith K. Kupinski, Russell A. Chipman, James B. Breckinridge

Jeffrey Davis, Meredith K. Kupinski, Russell A. Chipman, James B. Breckinridge, "HabEx polarization ray trace and aberration analysis," Proc. SPIE 10698, Space Telescopes and Instrumentation 2018: Optical, Infrared, and Millimeter Wave, 106983H (16 July 2018); doi: 10.1117/12.2313670

Event: SPIE Astronomical Telescopes + Instrumentation, 2018, Austin, Texas, United States 


\title{
HabEx Polarization Ray Trace and Aberration Analysis*
}

\author{
Jeffrey Davis ${ }^{\mathrm{a}}$, Meredith K. Kupinski ${ }^{\mathrm{a}}$, Russell A. Chipman ${ }^{\mathrm{a}}$, and James B. Breckinridge ${ }^{\mathrm{a}, \mathrm{b}}$ \\ ${ }^{a}$ College of Optical Sciences, The University of Arizona, Tucson, Arizona, 85721 USA \\ ${ }^{\text {b}}$ California Institute of Technology, Pasadena, CA, 91109 USA
}

\begin{abstract}
The flux difference between a terrestrial exoplanet and a much brighter nearby star creates an enormous optical design challenge for space-based imaging systems. Coronagraphs are designed to block the star's flux and obtain a high-dynamic-range image of the exoplanet. The contrast of an optical system is calculated using the point spread function (PSF). Contrast quantifies starlight suppression of an imaging system at a given separation of the two objects. Contrast requirements can be as small as $10^{-10}$ for earth-like planets. This work reports an analysis of the September 2017 Habitable Exoplanet Imaging Mission (HabEx) end-to-end optical system prescription for geometric and polarization aberrations across the 450 to $550 \mathrm{~nm}$ channel. The Lyot coronagraph was modeled with a vector vortex charge 6 mask but without adaptive optics (AO) to correct the phase of the Jones pupil. The detector plane irradiance was calculated for three states of the telescope/coronagraph system: (1) free of geometric and polarization aberrations; (2) isotropic mirror coatings throughout the end-toend system; and (3) isotropic mirrors with form birefringence on the primary mirror. For each of these three states the system response both with and without a coronagraph mask was calculated. Two merit functions were defined to quantify the system's ability to attenuate starlight: (1) normalized polychromatic irradiance (NPI), and (2) starlight suppression factor (SSF). Both of these are dimensionless and their values are functions of position across the focal plane. The NPI is defined as the irradiance point-by-point across the detector plane with a coronagraph mask divided by the value of the on-axis irradiance without a coronagraph mask. The SSF is the irradiance point-by-point across the detector plane with a coronagraph mask divided by the pointby-point value of the irradiance across the detector plane without a coronagraph mask. Both the NPI and the SSF provide insights into coronagraph performance. Deviations from the aberration-free case are calculated and summarized in table 2. The conclusions are: (1) the HabEx optical system is well-balanced for both geometric and polarization aberrations; (2) the spatially dependent polarization reflectivity for the HabEx primary mirror should be specified to ensure the coating is isotropic; (3) AO to correct the two orthogonal polarization-dependent wavefront errors is essential.
\end{abstract}

*Astronomical Telescopes and Instruments SPIE conference, Austin TX June 2018. Paper number 10698-120.

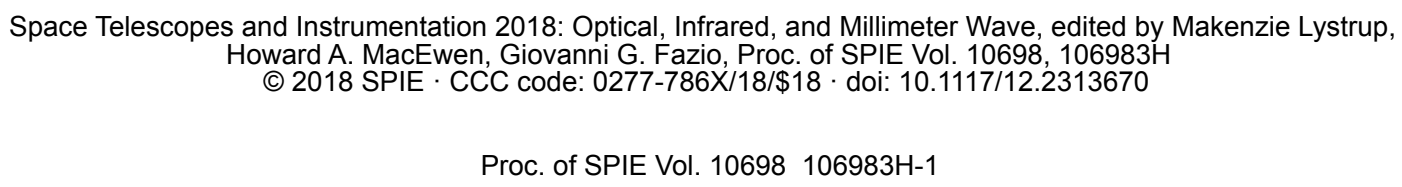

Proc. of SPIE Vol. $10698106983 \mathrm{H}-1$ 


\section{INTRODUCTION}

The flux difference between a terrestrial exoplanet and a much brighter nearby star creates an enormous optical design challenge for space-based imaging systems. Coronagraphs are designed to block the star's flux and obtain a high-dynamic-range image of the exoplanet. Current telescope/coronagraph systems have been optimized to be scalar-wave diffraction limited while polarization aberrations require vector-wave analysis. Instrument polarization limits coronagraph performance. This paper explores the sources responsible for polarization aberrations, describes the tools for characterizing polarization (vector-wave) aberrations, and compares performance metrics of a telescope/coronagraph system with and without polarization aberrations. The optical prescription for the Habitable Exoplanet Imaging Mission (HabEx) and a charge 6 vector vortex coronagraph are used to demonstrate the role of polarization aberrations in image formation. ${ }^{1}$ Table 1 lists the parameters of the telescope/coronagraph model. Three different cases of HabEx's optical design for the same basic optical prescription are analyzed: (1) aberration-free performance, (2) isotropic dielectric/metal mirrors (i.e no form birefringence), and (3) isotropic dielectric/metal mirrors with a form birefringent metal coating on the primary mirror (derived from birefringence measurements described in Section 2.2).

\begin{tabular}{|c|c|}
\hline Prescription Date & September 2017 \\
\hline Aperture & 4-m filled \\
\hline A/O & None (Flat mirrors) \\
\hline Coatings: Mirror 1 and 2 & $\mathrm{Al}+\mathrm{MgF}_{2}$ \\
\hline Coatings: Mirrors $3+$ & $\mathrm{Ag}+\mathrm{FSS} 99$ \\
\hline Mask & Vector Vortex Charge 6 \\
\hline Lyot Stop Size & $90 \%$ of the Entrance Pupil \\
\hline
\end{tabular}

Table 1: Telescope/coronagraph parameters used in the model

This paper is separated into six sections. Section 1 is the introduction. Section 2 describes sources of polarization aberrations. Section 3 discusses the steps in a polarization ray trace and the pertinent outputs from a ray trace. Section 4 describes the numerical methods used to model the coronagraph. Section 5 presents the results of the polarization ray trace of the HabEx optical system with a vector vortex charge 6 coronagraph. Section 6 provides our conclusions based on the results of the polarization ray trace.

Proc. of SPIE Vol. $10698106983 \mathrm{H}-2$ 


\section{SOURCES OF POLARIZATION ABERRATIONS}

\subsection{Thin Films}

Thin film coatings are deposited onto the surface of metallic mirrors to increase their reflectivity and to protect the mirror. The HabEx optical design uses two different coatings for its reflecting surfaces: (1) the primary and secondary mirrors use aluminum over-coated with $\mathrm{MgF}_{2},(2)$ the remaining reflecting surfaces use a more complicated thin film, FSS99, to coat silver. Figures 1a-1b show s- and p-polarized magnitude, $|\mathrm{r}|$, and phase, $\phi$, of the reflection coefficient for the over-coated $\mathrm{MgF}_{2}$, and figures 1c-1d show FSS99.

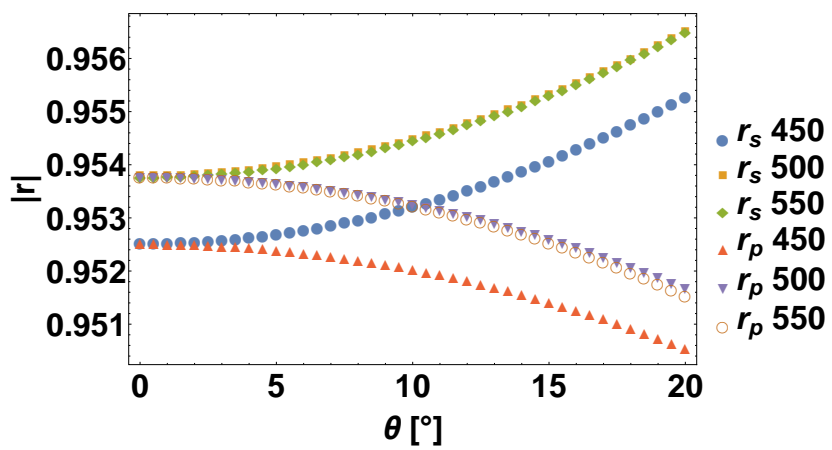

(a) Magnitude of reflection coefficient for $\mathrm{MgF}_{2}$

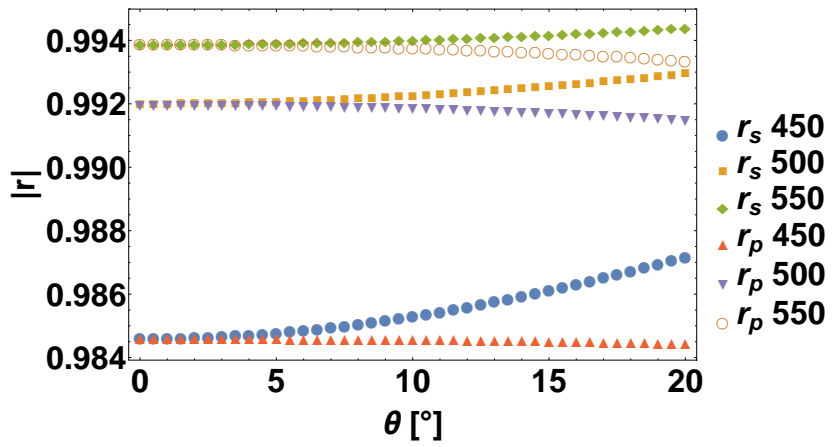

(c) Magnitude of reflection coefficient for FSS99

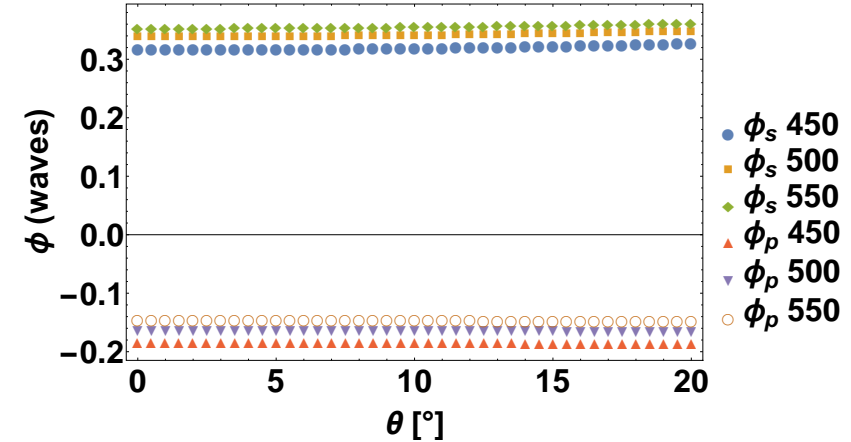

(b) Phase of reflection coefficient for $\mathrm{MgF}_{2}$

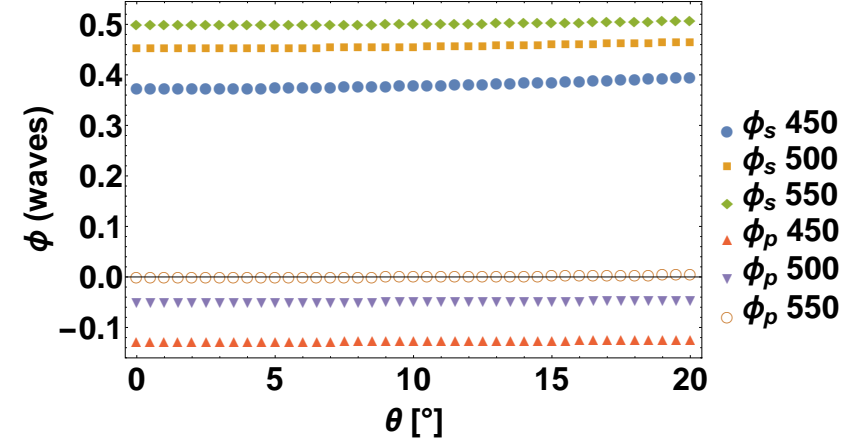

(d) Phase of reflection coefficient for FSS99

Figure 1: 450, 500, and $550 \mathrm{~nm}$ wavelengths reflection coefficient magnitude (a,c) and phase (b,d) vs. angle of incidence (in degrees) for s,p polarized light for $25 \mathrm{~nm} \mathrm{MgF}_{2}$ (a,b) on aluminum and FSS99 (c-d) on silver.

There are eleven reflecting surfaces, including the primary mirror, before HabEx's coronagraph mask plane and another four reflecting surfaces after the coronagraph mask plane. Each surface will attenuate and partially polarize the light, as shown in figures 1a - 1d. The maximum angles of incidence for the primary and secondary mirror are $11.8^{\circ}$ and $13.3^{\circ}$, respectively. The maximum angle of incidence for the other reflecting surfaces is $14.6^{\circ}$. Diattenuation is a measure of the transmittance or reflectance difference between s-polarization and 
p-polarization, and is defined as

$$
D=\frac{\left|r_{s}\right|^{2}-\left|r_{p}\right|^{2}}{\left|r_{s}\right|^{2}+\left|r_{p}\right|^{2}} .
$$

Retardance is the phase difference between s-polarization and p-polarization, $\Delta \phi=\phi_{s}-\phi_{p}$. The cumulative effect of each surface defines the diattenuation and retardance of the system. A common approach to reduce the diattenuation and retardance of an optical system is to choose a system layout that minimizes the angles of incidence at each surface. The polarization aberrations of a fold mirror can be balanced by aligning the s-polarization of the fold mirror with the p-polarization of a second fold mirror. ${ }^{2}$

\subsection{Form Birefringence}

Polarization aberrations in coronagraph systems have been studied assuming isotropic thin films. ${ }^{3}$ Here we investigate the role of anisotropic thin films. Form birefringence is refractive anisotropy due to the microstructure of the metal thin film. ${ }^{4}$ Mirror form birefringence can also contribute to polarization aberrations. For example, in the process of depositing a thin film coating of aluminum, the microscopic grain structure of the aluminum may be amorphous in certain regions and columnar in other regions. ${ }^{5}$ This columnar structure appears to be attributed to oxidation of the tiny vapor droplets as they adhere to the glass substrate. ${ }^{6}$ This anisotropy leads to regions of the mirror exhibiting spatially-varying retardance associated with the deposition process.

\section{POLARIZATION RAY TRACING HabEx}

\subsection{Overview}

Our polarization ray trace outputs polarization ray tracing (PRT) matrices for each ray traced through the system. $^{7}$ PRT matrices are three-dimensional extensions of Jones matrices and are used to calculate Jones pupils which are a $2 \times 2$ complex-valued Jones matrix at each spatial coordinate (x,y) in the pupil. The electric field in the entrance pupil (NP) is a Jones vector, denoted $\mathbf{E}_{N P}$, and in the exit pupil (XP), $\mathbf{E}_{X P}$. The Jones pupil describes the linear mapping of the polarization state of rays at the entrance pupil to the polarization state at the exit pupil, $\mathbf{E}_{X P}=\mathbf{J E}_{N P}$. The Jones pupil is dimensionless. The amplitude response matrix (ARM), as shown in figure $3 \mathrm{a}$, is defined as the two-dimensional spatial Fourier transform of each component $\left(i . e\right.$. $\mathbf{J}_{X X}$, $\mathbf{J}_{X Y}, \mathbf{J}_{Y X}$, and $\mathbf{J}_{Y Y}$ ) of the Jones pupil. ${ }^{8}$ The ARM is dimensionless. For an incident plane wave, the amplitude and phase of the electric field at the image plane are given by the matrix-vector multiplication of the ARM (a $2 \times 2$ complex-valued matrix) with the incident electric field (a $2 \times 1$ complex-valued vector). For unpolarized illumination, the sum of the squared modulus of each of the four components of the ARM yields the point spread function (PSF). More generally, the point spread matrix (PSM) describes the irradiance distribution and polarization for incoherent imaging. ${ }^{9}$ The PSM is a $4 \times 4$ Mueller matrix generalization of the PSF. ${ }^{8}$ The 
PSM is calculated by transforming the Jones matrices of the ARM into Mueller matrices. Merit functions for system performance are calculated from the PSF with and without a coronagraph and are compared with and without polarization calculations to quantify the effect of polarization aberrations. All polarization ray trace results presented here were calculated using Polaris-M, polarization ray tracing software from Airy Optics. ${ }^{10}$ Readers interested in the polarization ray tracing process are encouraged to read (R.A. Chipman, W. Lam, G. Yun $)^{11}$ or (G. Yun, K. Crabtree, R.A. Chipman). ${ }^{7}$ For examples of polarization ray tracing of optical systems, and in particular coronagraphs, see (J.B. Breckinridge, W.S.T. Lam, and R.A. Chipman). ${ }^{9}$

\subsection{Polarization Ray Trace Matrix (PRT)}

Polaris-M specifies polarization in global coordinates rather than local coordinates using PRTs. Given a threedimensional electric field before a surface, $\vec{E}_{q}$, with direction of propagation, $\hat{k}_{q}$, and an optical surface represented by the $3 \times 3$ PRT matrix, $\mathbf{P}_{q}$, the electric field and direction of propagation after interaction with the surface, $\vec{E}_{q+1}$ and $\hat{k}_{q+1}$, are described by $\vec{E}_{q+1}=\mathbf{P}_{q} \vec{E}_{q}$ and $\hat{k}_{q+1}=\mathbf{P}_{q} \hat{k}_{q}{ }^{7}$ The PRT matrix for reflection at the $q^{t h}$ optical surface in a system is given by

$$
\mathbf{P}_{q}=\mathbf{O}_{\text {out }, q} \mathbf{J}_{r, q} \mathbf{O}_{i n, q}^{-1}
$$

where

$$
\begin{gathered}
\mathbf{O}_{i n, q}^{-1}=\left(\begin{array}{ccc}
\hat{s}_{x, q} & \hat{s}_{y, q} & \hat{s}_{z, q} \\
\hat{p}_{x, q} & \hat{p}_{y, q} & \hat{p}_{z, q} \\
\hat{k}_{x, q} & \hat{k}_{y, q} & \hat{k}_{z, q}
\end{array}\right), \mathbf{O}_{\text {out }, q}=\left(\begin{array}{ccc}
\hat{s}_{x, q} & \hat{p}_{x, q}^{\prime} & \hat{k}_{x, q+1} \\
\hat{s}_{y, q} & \hat{p}_{y, q}^{\prime} & \hat{k}_{y, q+1} \\
\hat{s}_{z, q} & \hat{p}_{z, q}^{\prime} & \hat{k}_{z, q+1}
\end{array}\right), \mathbf{J}_{r, q}=\left(\begin{array}{ccc}
\left|r_{s}\right| e^{i \phi_{s}} & 0 & 0 \\
0 & \left|r_{p}\right| e^{i \phi_{p}} & 0 \\
0 & 0 & 1
\end{array}\right) \\
\hat{s}_{q}=\frac{\hat{k}_{q} \times \hat{k}_{q+1}}{\left|\hat{k}_{q} \times \hat{k}_{q+1}\right|}, \quad \hat{p}_{q}=\hat{k}_{q} \times \hat{s}_{q}, \quad \hat{p}_{q}^{\prime}=\hat{k}_{q+1} \times \hat{s}_{q} .
\end{gathered}
$$

Subscripts $x, y$, and $z$ refer to projections onto the $x, y$, and $z$ axes. $\mathbf{O}_{i n, q}^{-1}$ rotates the incident electric field from global coordinates into the local $(\hat{s}, \hat{p}, \hat{k})$ coordinate system defined by the incoming and reflected directions of propagation. $\mathbf{J}_{r, q}$ is the Jones matrix of the surface in the s-p basis. $\mathbf{O}_{\text {out }, q}$ rotates the resulting electric field back into global coordinates after reflection. Each optical surface in the optical system has a corresponding PRT, and the entire system with $Q$ optical surfaces is represented by the matrix multiplication of every surfaces' PRT:

$$
\mathbf{P}_{\text {total }}=\mathbf{P}_{Q} \mathbf{P}_{Q-1} \ldots \mathbf{P}_{2} \mathbf{P}_{1}=\prod_{q=Q}^{1} \mathbf{P}_{q}
$$




\subsection{Jones Pupil and Amplitude Response Matrix (ARM)}

To compute the Jones matrix for each ray, the cumulative PRT matrix from Eq. 3 is used with Eq. 2. When the cumulative PRT matrix is used, $\mathbf{O}_{\text {in }}$ and $\mathbf{O}_{\text {out }}$ are defined by the $\hat{s}, \hat{p}, \hat{k}$ basis entering and exiting the system. The Jones pupil is computed from $\mathbf{O}_{\mathbf{i n}}, \mathbf{O}_{\mathbf{o u t}}$, and the PRT for each ray traced through the system. Eigenvalue decomposition is applied to each Jones matrix that makes up the Jones pupil. The difference of the phase of the eigenvalues is the retardance while the diattenuation is calculated from amplitudes of the eigenvalues by Eq. 1 . Figure 2 shows the Jones pupil of the fore-optics before the HabEx coronagraph for $500 \mathrm{~nm}$ light. The variations of the Jones pupil amplitude and phase arise from the variations of angle and plane of incidence across each of the 11 reflecting surfaces (see figures 1a-1d). To compare, an aberration-free, diffraction-limited Jones pupil would be an identity matrix inside the pupil, i.e. the off-diagonal elements would have zero amplitude and phase.
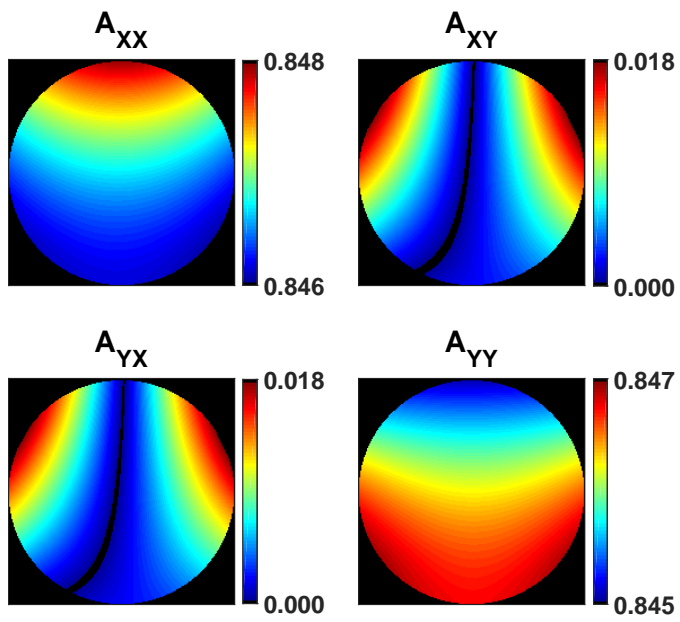
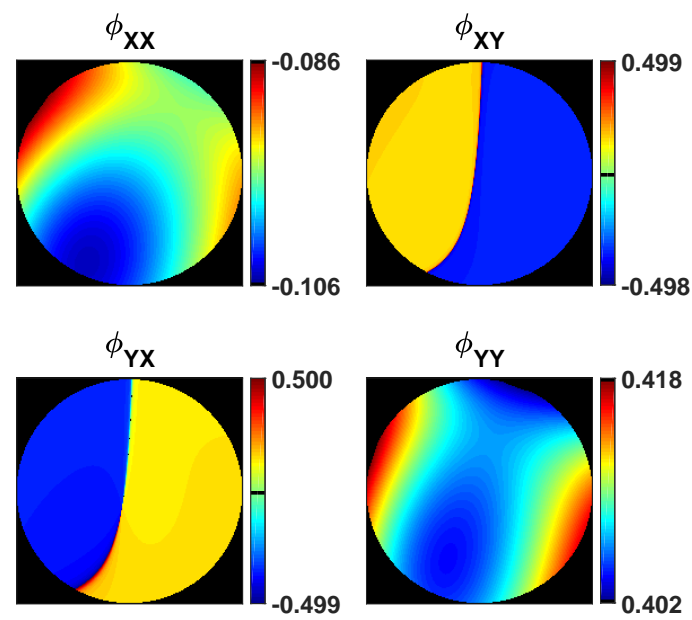

(a) Amplitude of Jones Pupil

(b) Phase of Jones Pupil

Figure 2: For Habex's fore-optics at $500 \mathrm{~nm}$ the Jones pupil: (a) amplitude and (b) phase in waves.

The off-diagonal elements of figure $2 \mathrm{a}$ are close to zero, indicating that the system is well-corrected for cross polarization leakage. The on-diagonal elements are similar, differing in amplitude at most by about three tenths of a percent indicating that slightly more (tenths of a percent) x-polarized light is incident on the coronagraph than y-polarized light. The discontinuities in the phase of the off-diagonals of figure $2 \mathrm{~b}$ are $\pi$ phase shifts that occur when the amplitude linearly passes through a zero. A small amount of astigmatism is seen in the phase of the on-diagonal elements. 

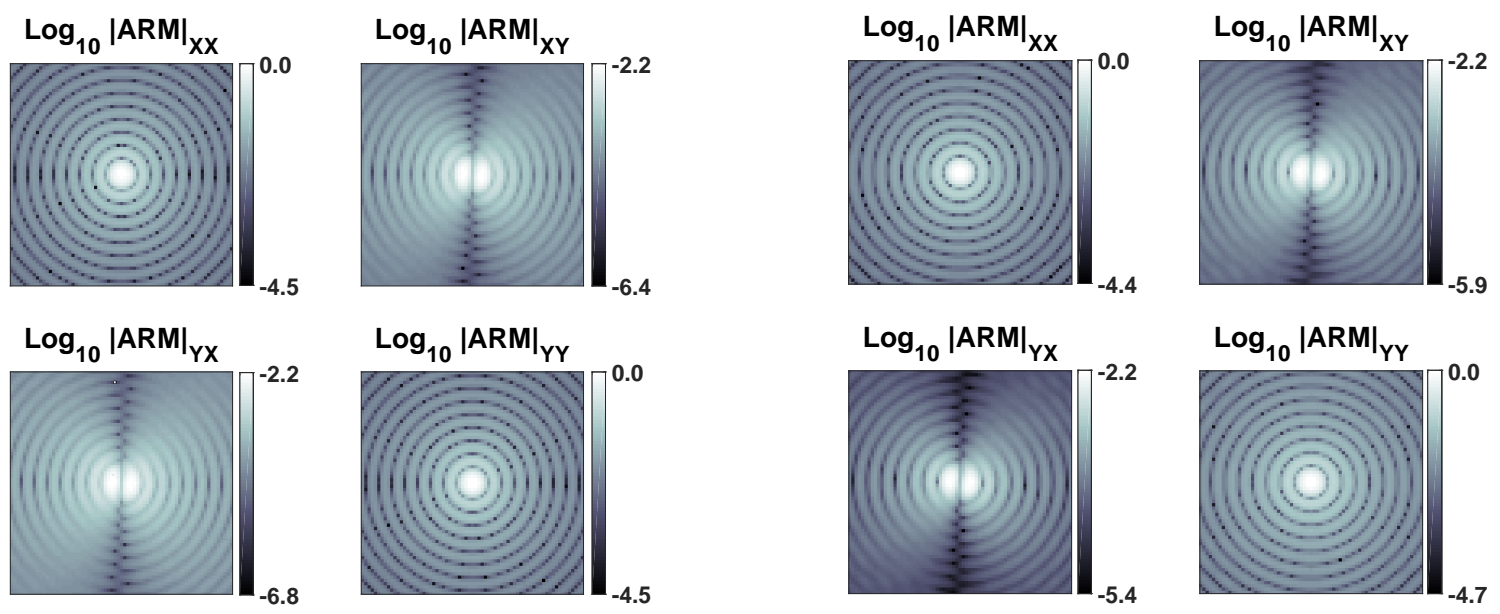

(a) $\log _{10}|\mathbf{A R M}|$ of fore-optics for isotropic case

(b) $\log _{10}|\mathbf{A R M}|$ of fore-optics for birefringent case

Figure 3: $\log _{10}|\mathbf{A R M}|$ at the focal plane immediately before the coronagraph mask for (a) isotropic metal/dielectric coatings on every surface and (b) isotropic metal/dielectric coatings on every surface and birefringence map applied to the primary mirror.

Figure 3a (left) shows the $\log _{10}|\mathbf{A R M}|$ at the focal plane directly in front of the coronagraph mask that is calculated from the Jones pupil in figure 2. Figure 3b (right) shows the $\log _{10}|\mathbf{A R M}|$ at the focal plane directly in front of the coronagraph mask that is calculated with birefringence on the primary mirror. The on-diagonal isotropic $\log _{10}|\mathbf{A R M}|$ elements are similar to an Airy pattern but slightly broadened. Adding in birefringence to the primary broadens the $\log _{10}|\mathbf{A R M}|$ even further, although this difference is not discernible in figure 3a. The maximum values of the off-diagonal elements, as compared to the diagonal elements, are 2.5-orders of magnitude lower. Non-zero off-diagonal elements indicate the presence of polarization aberrations. These terms are commonly referred to as polarization leakage because they describe light which is transformed into an orthogonal polarization state between input and output. The two off-diagonal terms both show a bifurcated structure that is not maximum at the center and is approximately symmetric about a vertical line of symmetry. The separation between the peaks of this bifurcated structure is approximately 17 milliarcseconds.

\subsection{Form Birefringent Map Applied to HabEx Primary}

The coating geometry used to deposit large area primary mirrors $\left(>12 m^{2}\right)$ is different than that used for smaller mirrors $\left(<2 m^{2}\right)$. The spatial dependence of the polarization reflectivity of a bare aluminum 3.75-meter diameter test sphere was measured at the University of Arizona Mirror Lab by Daugherty (2018). ${ }^{12}$ This mirror is used for optical wavefront testing of the 8-meter GMT primary mirrors. The bare aluminum was deposited using 
the same chamber and processes employed to coat the primary mirror for the Mayall 4-meter telescope at Kitt Peak National Observatory. The coating shows a spatial dependence of the polarization reflectivity caused by form birefringence. ${ }^{12}$ Figure 4 shows the Jones matrix associated with the form birefringence. To understand the potential role of form-birefringence on the primary mirror, we scaled and applied this form birefringence measurement onto the primary mirror of HabEx.
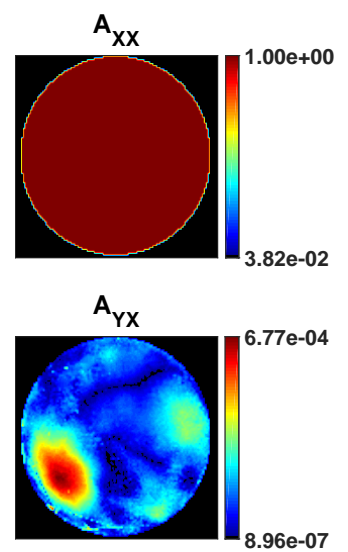
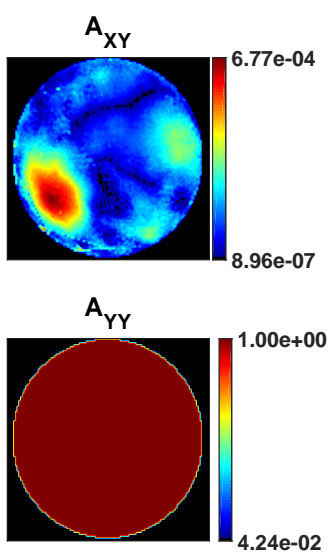
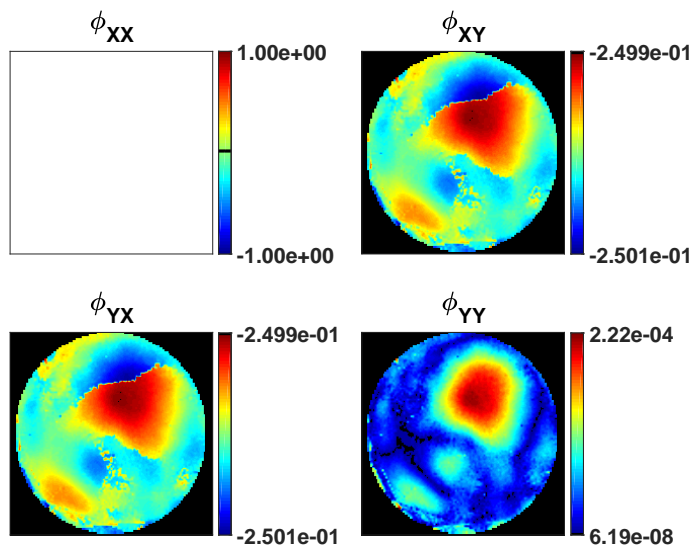

(a) Amplitude

(b) Phase [waves]

Figure 4: Jones matrix amplitude (a) and phase in waves (b) of the birefringence map of the 3.75-meter diameter test sphere. The phase of the XX component is zero across the entire map due to convention in the conversion of the birefringence map from a Mueller matrix into a Jones matrix.

The HabEx flight mirror coating design, processing, and deposition geometry will differ from the 3.75-m mirror, but these calculations show the potential of form birefringence to degrade coronagraph performance. Daugherty (2018) is the first to use an innovative polarization metrology technique to measure the form birefringence of a large area mirror. This work is the first application of a form birefringence measurement to the analysis of starlight suppression suggesting that high-contrast imaging requires a form birefringence coating requirement.

\section{NUMERICAL METHODS FOR MODELING CORONAGRAPHS}

\subsection{Overview}

Ray tracing by itself does not characterize vector-wave phenomena like interference and diffraction. Accurate polarization image formation is calculated using polarization ray tracing from the entrance to exit pupil and then Fourier optics to diffract the vector field to the image plane. Modeling the effects of a coronagraph on an electric field requires vector diffraction optics algorithms. Stellar coronagraphs are optical systems designed to reject 
direct light from a star so that objects near the star can be observed. Several types of coronagraphs include (but are not limited to): band-limited coronagraphs, ${ }^{13}$ phase-mask coronagraphs,${ }^{14}$ and optical vortex coronagraphs. ${ }^{15}$ The coronagraph analyzed in this work uses a vector vortex mask analyzed by pupil decomposition and Fourier analysis using the semi-analytic method ${ }^{16}$ as described below. Pupil decomposition is not specific to HabEx and is applicable to any compound optical system.

\subsection{Pupil Decomposition}

Consider an optical system divided into two separate subsystems, system A and system B, where the exit pupil of $\mathrm{A}$ is the entrance pupil of $\mathrm{B}$. The coronagraph mask is located at an intermediate image plane between $\mathrm{A}$ and B. System A encompasses all the optics up to (but not including) the coronagraph mask, and system B encompasses all the optics after the coronagraph mask. The advantage of this pupil decomposition is that the Jones pupils of each subsystem (denoted $\mathbf{J} \mathbf{P}_{A}$ and $\mathbf{J P} \mathbf{P}_{B}$ ) can be computed from ray traces which do not need to be repeated for varying coronagraph mask designs. Figure 2 shows $\mathbf{J P}_{A}$ at $500 \mathrm{~nm}$.

\subsection{Modeling a Vector Vortex Coronagraph}

The ARM of system A describes the electric field distribution at the coronagraph mask plane as a function of incident polarization state. The electric field is attenuated by the coronagraph which is modeled by an element-by-element multiplication of the ARM with the coronagraph mask Jones matrix. The vector vortex is a half-wave retarder with a continuously variable fast-axis which creates a polarization vortex. Vector vortices can create azimuthal and radial polarization patterns when illuminated with uniform linear polarized light. ${ }^{17}$ The Jones matrix for an ideal vector vortex is real-valued and given by ${ }^{18}$

$$
\mathbf{V V}_{m}(x, y)=\left[\begin{array}{cc}
\cos \left(m \tan ^{-1}(y / x)\right. & \sin \left(m \tan ^{-1}(y / x)\right) \\
\sin \left(m \tan ^{-1}(y / x)\right. & -\cos \left(m \tan ^{-1}(y / x)\right.
\end{array}\right]
$$

where $x$ and $y$ are spatial coordinates in the coronagraph mask plane and $m$ is the charge (index) of the vector vortex. Figure 5a shows the Jones matrix for the charge 6 vector vortex mask we use to model HabEx. 

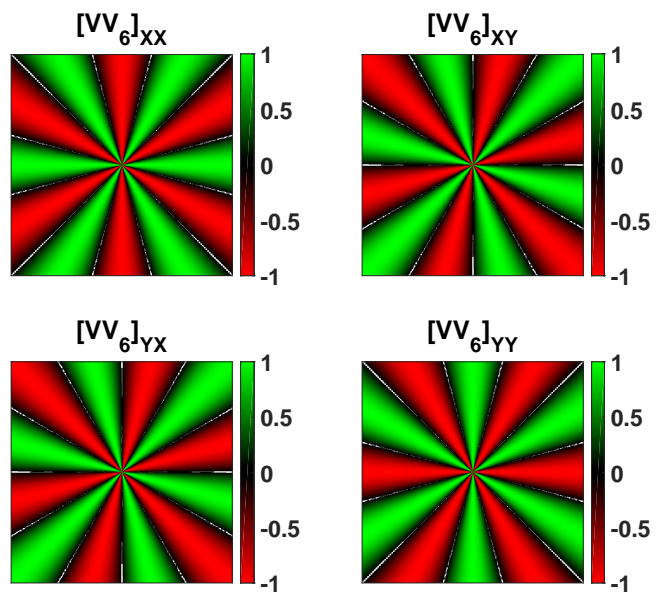

(a) Jones matrix of $\mathbf{V} \mathbf{V}_{6}$

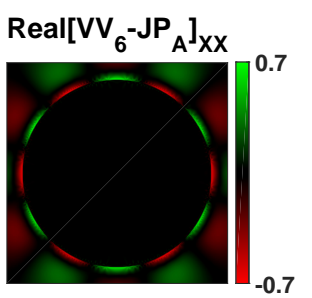

Real[VV $\left.{ }_{6}-\mathrm{JP}_{\mathrm{A}}\right]_{\mathrm{YX}}$
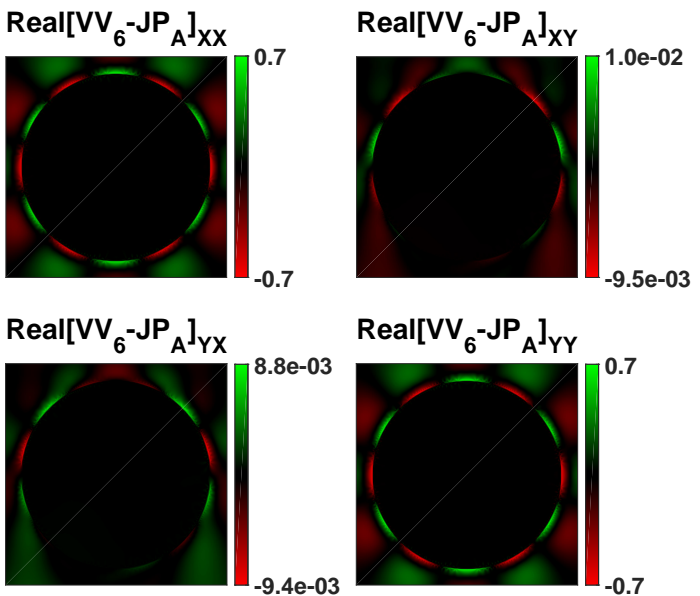

Real $\left[V_{6}-J_{A}\right]_{Y Y}$

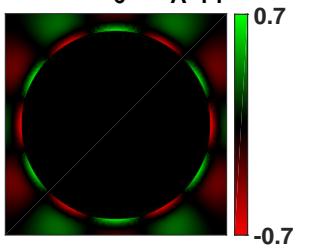

(b) Real part of $\mathbf{V} \mathbf{V}_{6}-\mathbf{J P} \mathbf{P}_{A}$

Figure 5: (a) Jones matrix of a vector vortex coronagraph with charge $m=6$ (denoted $\mathbf{V} \mathbf{V}_{6}$ ) as defined in Eq. 4 and (b) Real part of the Jones Pupil of Part A with $\mathbf{V} \mathbf{V}_{6}$ (denoted $\mathbf{V} \mathbf{V}_{6}-\mathbf{J P}_{A}$ ) at $500 \mathrm{~nm}$.

Careful consideration of the vortex plane sampling is necessary because the vector vortex spatial frequency increases towards the center of the vortex. One method to handle these higher spatial frequency variations, the semi-analytic method, was developed for coronagraph applications. ${ }^{16}$ This algorithm applies two Discrete Fourier Transform (DFT) operations: (1) a DFT that highly samples the central region of the ARM attenuated by the vector vortex, and (2) a DFT that more coarsely samples the entire attenuated ARM. These two DFTs are then stitched together in the pupil plane via a windowing algorithm to yield $\mathbf{V} \mathbf{V}_{6^{-}} \mathbf{-} \mathbf{J P}_{A}$ as seen in figure $5 \mathrm{~b}$. Matrix multiplication of $\mathbf{V V}_{6}-\mathbf{J} \mathbf{P}_{A}$ with $\mathbf{J P}_{B}$ yields the Jones pupil for the entire system including the vector vortex of charge 6 .

\section{RESULTS}

\subsection{System Performance Metric Definitions}

The spatially-varying irradiance at the detector plane depends upon the polarization state of the source. For an incoherent source, the irradiance is the sum of the four PSF components. A linear polarizer at the input and another linear polarizer at the output of the system can be oriented in the X- or Y-direction to probe each of the four PSF components. There are four orientations for these pair of polarizers in which they are either aligned (XX,YY) or orthogonal (XY, YX). From each of these four polarizer orientations the polarizations components (XX, YY, XY, YX) can be measured. At each orientation of the pair of polarizers the monochromatic irradiance 
at the detector plane is denoted

$$
\mathbf{I}(x, y ; \lambda)=\left[\begin{array}{ll}
\mathrm{I}_{X X}(x, y ; \lambda) & \mathrm{I}_{X Y}(x, y ; \lambda) \\
\mathrm{I}_{Y X}(x, y ; \lambda) & \mathrm{I}_{Y Y}(x, y ; \lambda)
\end{array}\right] .
$$

Here $\mathbf{I}(x, y)$ is a $2 \times 2$ real-valued matrix in units of $\mathrm{W} / \mathrm{m}^{2}$.

Exoplanet coronagraphs observe white-light thermal sources which requires a broad optical bandwidth to obtain sufficient power for detection. To simulate polychromatic image formation, the monochromatic irradiance is spectrally sampled and summed

$$
\mathbf{I}(x, y)=\frac{1}{K} \sum_{k=1}^{K} \mathbf{I}\left(x, y ; \lambda_{k}\right) .
$$

Here $\lambda_{k}$ is a wavelength and $K$ is the number of wavelengths that are sampled. The incoherent sum over polarization components in the unpolarized case is denoted by a tilde over the irradiance

$$
\tilde{\mathrm{I}}(x, y)=\mathrm{I}_{X X}(x, y)+\mathrm{I}_{Y Y}(x, y)+\mathrm{I}_{X Y}(x, y)+\mathrm{I}_{Y X}(x, y) .
$$

To quantify the performance of the coronagraph mask, Eq. 7 is computed twice. Once with the mask $(M)$ and once open $(O)$ and denoted $\tilde{\mathrm{I}}^{M}(x, y)$ and $\tilde{\mathrm{I}}^{O}(x, y)$, respectively.

The normalized polychromatic irradiance (NPI) is defined as a dimensionless metric given by

$$
\mathrm{NPI}(x, y)=\frac{\tilde{\mathrm{I}}^{M}(x, y)}{\tilde{\mathrm{I}}^{O}(0,0)}=\frac{\mathrm{I}_{X X}^{M}(x, y)}{\tilde{\mathrm{I}}^{O}(0,0)}+\frac{\mathrm{I}_{X Y}^{M}(x, y)}{\tilde{\mathrm{I}}^{O}(0,0)}+\frac{\mathrm{I}_{Y X}^{M}(x, y)}{\tilde{\mathrm{I}}^{O}(0,0)}+\frac{\mathrm{I}_{Y Y}^{M}(x, y)}{\tilde{\mathrm{I}}^{O}(0,0)}
$$

where $\operatorname{NPI}(x, y)$ is a real-valued scalar across the detector plane and $\tilde{\mathrm{I}}^{O}(0,0)$ is the on-axis incoherent polychromatic irradiance without a coronagraph mask. The NPI shows the shape of the irradiance pattern at the detector plane.

The starlight suppression factor (SSF) is also a dimensionless metric defined as the quotient of $\tilde{\mathrm{I}}^{M}(x, y)$ to $\tilde{\mathrm{I}}^{O}(x, y)$

$$
\operatorname{SSF}(x, y)=\frac{\tilde{\mathrm{I}}^{M}(x, y)}{\tilde{\mathrm{I}}^{O}(x, y)} .
$$

SSF is real-valued at every point in the detector plane. Note that the numerator of the NPI (Eq. 8) and the SSF (Eq. 9) are the same but the denominators are not. The SSF's denominator is dependent on the position in the irradiance pattern while the denominator of the NPI is a constant scalar-value. The SSF describes how efficiently the vector vortex removes the starlight at any point in the detector plane. Smaller values of SSF indicate that less starlight is allowed to reach the detector while larger values of SSF indicate that more starlight is allowed 
through to the detector at that point. Normalized polychromatic irradiances are shown in figures $7 \mathrm{a}-7 \mathrm{c}$ and 8 , and SSF are shown in figures $7 \mathrm{~d}-7 \mathrm{f}$ and 9.

The following sections show NPI and SSF plots for several HabEx models: (1) aberration-free, diffractionlimited performance, (2) isotropic dielectric/metal mirrors (i.e. no birefringence), and (3) isotropic dielectric/metal mirrors with a form birefringent anisotropic metal coating on the primary mirror. The HabEx flight mirror coating design, processing, and deposition geometry will differ from the $3.75-\mathrm{m}$ mirror, but these calculations show the potential of form birefringence to degrade coronagraph performance.

Polychromatic images are computed with, $K=18$ and $\lambda_{k}=[450,453,456,460,469,475,478,492,500,503$, $510,517,525,528,535,540,546,550] \mathrm{nm}$ (see Eq. 6). Non-uniform wavelength spacing was found to reduce artifacts compared to the summation of equally spaced wavelengths.

\subsection{Images of the Performance Metrics}

Figure 6a shows the four NPI terms in Eq. 8 for the case of a zero-polarization contribution from each of the TBD mirrors in the HabEx telescope/coronagraph end-to-end system with the $\mathbf{V V}_{6}$ mask in the coronagraph. We see that the off-diagonal elements are zero, which is characteristic of zero polarization aberrations. Also, we note that the on-diagonal elements exhibit point symmetry and are nearly invariant to azimuth angle.

Figure $6 \mathrm{~b}$ shows the four NPI terms in Eq. 8 for the case of all of the HabEx mirrors with isotropic coatings. Note that there is both power and structure in the images of the off-diagonal terms where we see a bifurcation about a vertical line of symmetry. All four images are represented on the same intensity scale.

Figure $6 \mathrm{c}$ shows the four NPI for the case where the HabEx primary mirror exhibits anisotropic form birefringence (shown in figure 3b) and the remaining mirrors are have isotropic polarization properties. The anisotropic primary mirror results in an approximately uniform increase for the on-diagonal images and negligible changes in the structure of the off-diagonal images.

Figures 7a-7f shows NPI and SSF images for HabEx with $\mathbf{V V}_{6}$. 

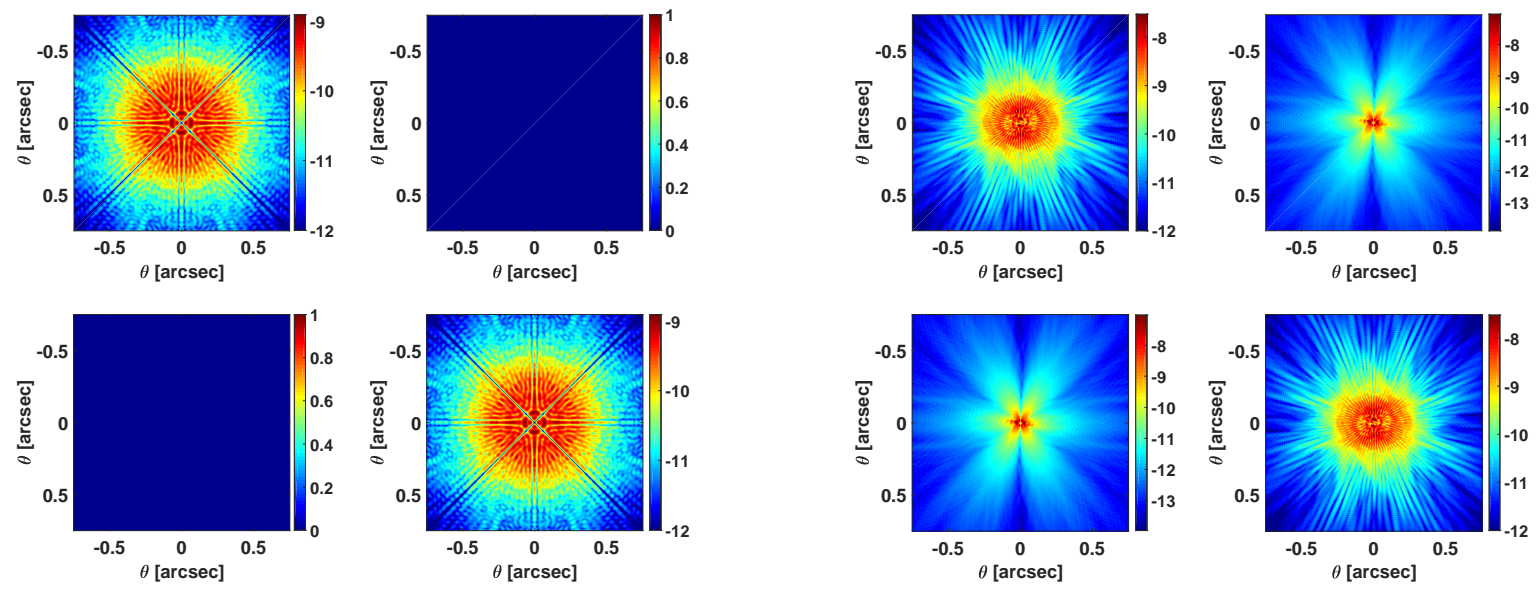

(a) Aberration-free

(b) Isotropic mirrors
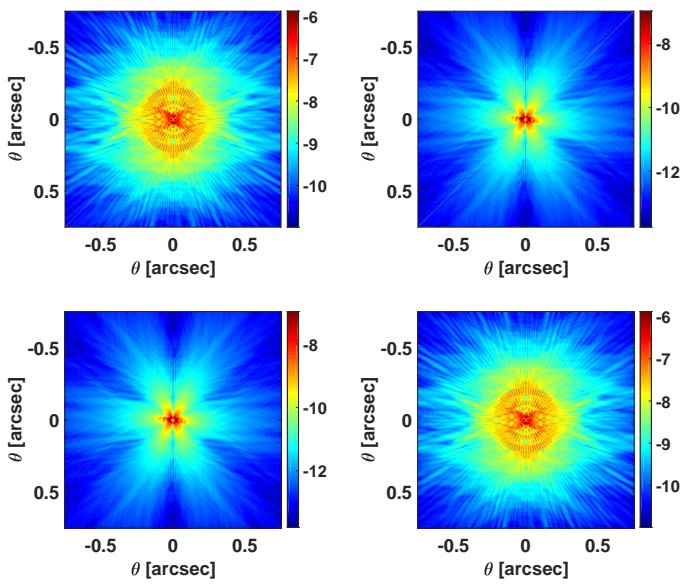

(c) Birefringence on primary

Figure 6: $\log _{10}$ of four NPI terms given in equation 8 for the detector plane (with $\mathbf{V} \mathbf{V}_{6}$ for the HabEx telescope/coronagraph system for three cases: (a) the aberration-free case, (b) isotropic coatings on every mirror, and (c) isotropic coatings on every mirror and the form birefringence map applied to the primary mirror. 


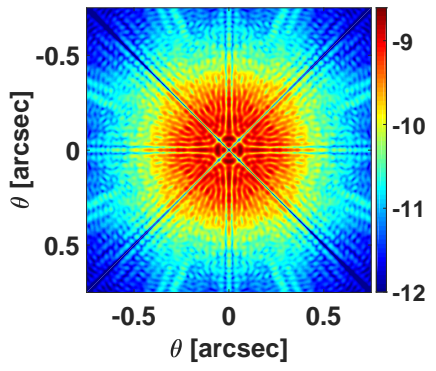

(a) NPI: Aberration-free

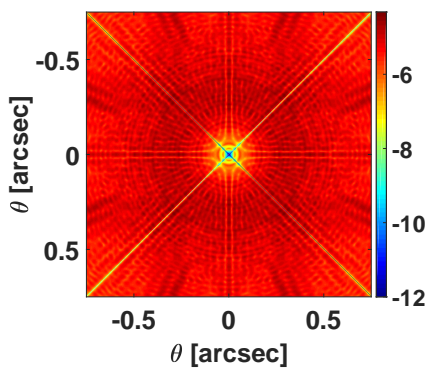

(d) SSF: Aberration-free

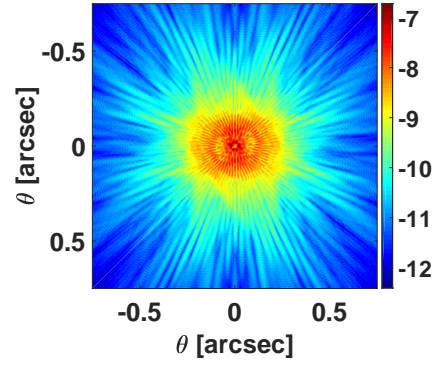

(b) NPI: Isotropic mirrors

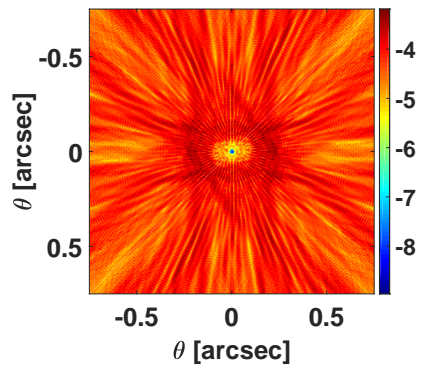

(e) SSF: Isotropic mirrors

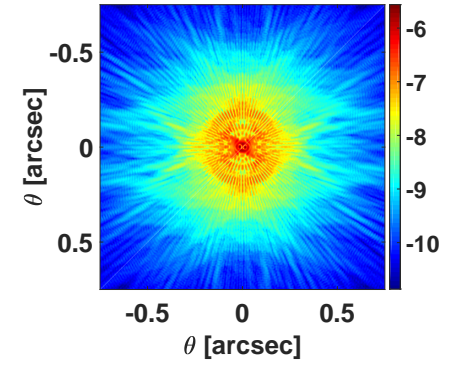

(c) NPI: Birefringence on primary

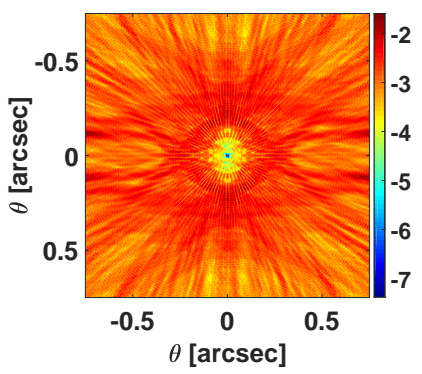

(f) SSF: Birefringence on primary

Figure 7: $\log _{10}$ NPI (top row) and $\log _{10} S S F$ (bottom row) images at the detector for (a,d) the aberrationfree case, $(b, e)$ isotropic coatings on every mirror, and $(c, f)$ isotropic coatings on every mirror and the form birefringence map applied to the primary mirror.

\subsection{Cross-sectional Slices of the Performance Metrics}

Figures $8 \mathrm{a}-8 \mathrm{~b}$ show horizontal and vertical slices through the NPI for the aberration-free, isotropic, and form birefringent primary cases. The inner working angle (IWA) of the HabEx coronagraph is 58 milliarcseconds (mas) (2.5 $\frac{\lambda}{d} @ 450 \mathrm{~nm}$ ), and the outer working angle (OWA) is 740 mas (32 $\left.\frac{\lambda}{d} @ 450 \mathrm{~nm}\right) .{ }^{19}$ These angles are represented by the dashed lines in figures $8 \mathrm{a}-8 \mathrm{~b}$. Figures $9 \mathrm{a}-9 \mathrm{~b}$ show horizontal and vertical slices through the SSF for the aberration-free, isotropic, and form birefringent primary cases. The mean NPI values and SSF values between the IWA and OWA for the three cases are shown in table 2 . 


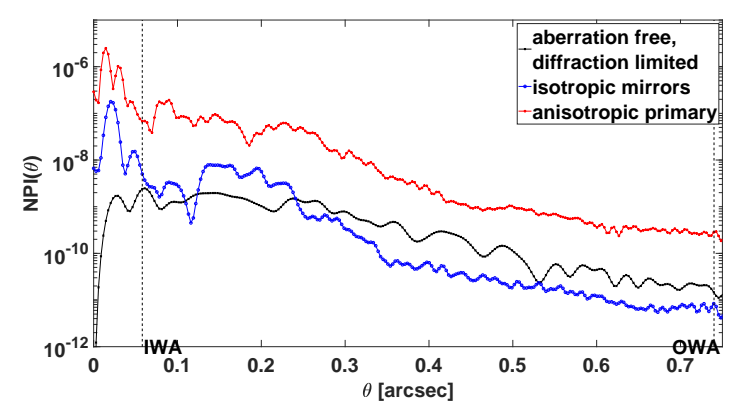

(a) Horizontal NPI cross-sectional slices

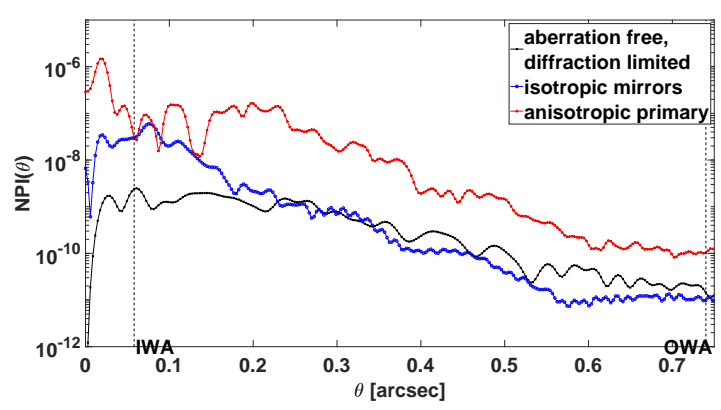

(b) Vertical NPI cross-sectional slices

Figure 8: Normalized polychromatic irradiance (NPI) as a function of field angle at the detector plane using the $\mathbf{V V}_{6}$ mask is shown for slices in the horizontal $(\mathrm{y}=0)$ direction (a) and the vertical $(\mathrm{x}=0)$ direction (b) for the aberration-free case (black lines), the aberration case for isotropic mirrors (blue) and the aberration case where the primary mirror has the birefringence map shown (red). Field angles are from 0 to 0.75 arc second. Inner (58 mas) and outer (740 mas) working angles are shown.

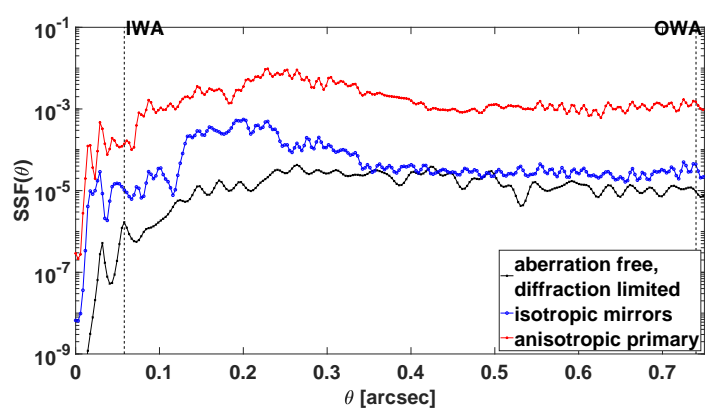

(a) Horizontal SFF cross-sectional slices

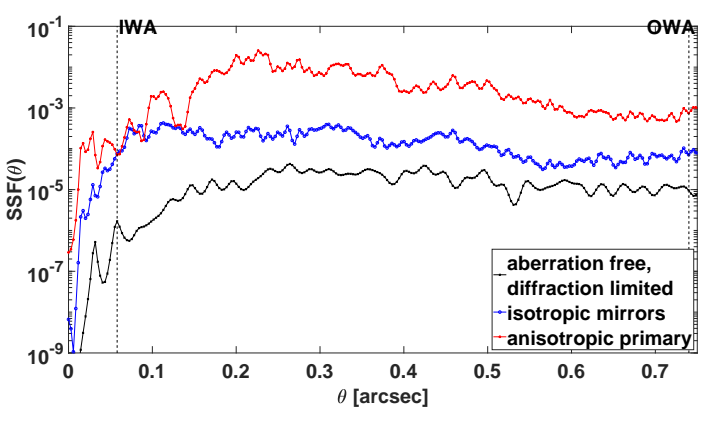

(b) Vertical SFF cross-sectional slices

Figure 9: Starlight suppression factor (SSF) as a function of field angle at the detector plane using the $\mathbf{V V}_{6}$ mask is shown for slices in the horizontal $(\mathrm{y}=0)$ direction $(\mathrm{a})$ and the vertical $(\mathrm{x}=0)$ direction $(\mathrm{b})$ for the aberration free case (black lines), the aberration case for isotropic mirrors (blue) and the aberration case where the primary mirror has the birefringence map shown (red). Field angles are from 0 to 0.75 arc second. Inner (58 mas) and outer (740 mas) working angles are shown. 


\begin{tabular}{|c|c|c|c|}
\hline & Aberration-Free & Isotropic Mirrors & Form Birefringent Primary \\
\hline $\mathrm{NPI}_{\text {horizontal }}$ & $5.8 \times 10^{-10}$ & $1.2 \times 10^{-9}$ & $2.3 \times 10^{-8}$ \\
\hline $\mathrm{NPI}_{\text {vertical }}$ & $5.8 \times 10^{-10}$ & $4.2 \times 10^{-9}$ & $3.1 \times 10^{-8}$ \\
\hline$\frac{\mathrm{NPI}_{\text {horizontal }}+\mathrm{NPI}_{\text {vertical }}}{2}$ & $5.8 \times 10^{-10}$ & $2.7 \times 10^{-9}$ & $2.7 \times 10^{-8}$ \\
\hline $\mathrm{SSF}_{\text {horizontal }}$ & $1.6 \times 10^{-5}$ & $9.1 \times 10^{-5}$ & $2.2 \times 10^{-3}$ \\
\hline $\mathrm{SSF}_{\text {vertical }}$ & $1.6 \times 10^{-5}$ & $1.7 \times 10^{-4}$ & $4.6 \times 10^{-3}$ \\
\hline$\frac{\mathrm{SSF}_{\text {horizontal }}+\mathrm{SSF}_{\text {vertical }}}{2}$ & $1.6 \times 10^{-5}$ & $1.3 \times 10^{-4}$ & $3.4 \times 10^{-3}$ \\
\hline
\end{tabular}

Table 2: Mean values for the horizontal and vertical slices between the IWA and OWA detector plane field points for both the normalized polychromatic irradiance (NPI) and starlight suppression factor (SSF) are tabulated. Column 1 describes the NPI and SSF factors given, column 2 shows values for the aberration-free case, column 3 shows values for all Habex mirrors coated isotropically and column 4 shows values assuming that only the HabEx primary mirror exhibits the form birefringence shown in figure 4, whereas the remaining mirrors are isotropic.

The field of view of interest for imaging exoplanets is an annulus between the IWA of 58 mas and the OWA of 740 mas. In figures 8, the black curve represents the NPI for aberration-free HabEx with the $\mathbf{V V}_{6}$ mask. NPI varies from $10^{-9}$ from 50 to about 300 mas and then drops to $10^{-10}$ near 400 mas and slopes down to $10^{-11}$ at the OWA. If we assume that all mirrors in HabEx are isotropic, then the NPI is nearly equal to the unaberrated case from the IWA to about 120 mas. At field points from 120 to about 200 mas the NPI for the isotropic mirrors are about a factor of 10 higher or $10^{-8}$. The NPI slopes down from $10^{-9}$ at 250 mas to become $10^{-10}$ for FOV values greater than 500 mas. The NPI for the case of the form birefringence on the primary shown in red in figure 8 , is $10^{-7}$ from the IWA to about $10^{-8}$ near 280 mas and then slopes down to become $10^{-9}$ at the OWA. Form birefringence on the primary mirror is seen to increase the NPI by almost 2 orders of magnitude between the IWA and the OWA for the $\mathrm{y}=0$ slice shown in figure $8 \mathrm{a}$. Figure $8 \mathrm{~b}$ shows curves similar to those in figure $8 \mathrm{a}$, but for the NPI along a slice in the $\mathrm{y}$-direction $(\mathrm{x}=0)$. The aberration-free NPI as a function of field angle is the same in both figures, indicating that it is rotationally symmetric. If we compare the shape of the NPI as a function of FOV shown in $8 \mathrm{a}$ with that shown in 8b NPI for the case where all the HabEx mirrors are isotropic we see that the NPI as a function of FOV is not rotationally symmetric which indicates that polarization aberrations are present.

Figure 9 shows the starlight suppression factor as a function of FOV from the IWA to the OWA. These are slices through a map of the pixel by pixel starlight suppression factor and show how well the $\mathbf{V V}_{6}$ mask attenuates starlight at each pixel in the FOV from 0 to 0.75 arc seconds. Figures $9 \mathrm{a}$ and $9 \mathrm{~b}$ show that the 
starlight suppression achieved by the $\mathbf{V} \mathbf{V}_{6}$ mask in the HabEx coronagraph interfaced to a fore-optic that contains a primary mirror with the form birefringence is two orders of magnitude less than for a homogeneous or isotropic primary mirror. Clearly, the project needs to specify that the primary mirror exhibit very low levels of form birefringence in the coating.

As isotropic and anisotropic polarization considerations are added to the model, the mean values of the NPI and SSF tend to increase. Adding in isotropic polarization properties to the model degrades the mean NPI by about a factor of 4.6 from the aberration-free case. Adding in form birefringence to the primary in addition to isotropic polarization properties to the model degrades the mean NPI by about about a factor of 46 from the aberration-free case, an order of magnitude more than the isotropic case. Adding in isotropic polarization properties to the model degrades the mean SSF by about a factor of 7.9 from the aberration-free case. Adding in form birefringence to the primary in addition to isotropic polarization properties degrades the mean SSF by about a factor of 210 from the aberration-free case.

\section{CONCLUSIONS}

Polarization aberrations arise from differences in Fresnel reflection coefficients between s-polarized and p-polarized at each metal mirrors' surface as well an form birefringence in the coating of metal mirrors. These amplitude and phase differences and non-uniformities cause the contrast of a coronagraph system to deviate from ideal. Using the outputs of a Polaris-M polarization ray trace of HabEx without AO as an example system, the NPI with isotropic polarization aberrations is degraded by about a factor of 4.6 from the aberration-free case and the SSF is degraded by about a factor of 7.9 . When a form birefringence measurement ${ }^{12}$ is added to the primary mirror, the NPI is degraded by about a factor of 46 from the aberration-free case and the SSF is degraded by about a factor of 210. These results highlight the necessity of form-birefringence coating requirements for flight mirrors to ensure there is no degradation in the telescope's ability to do high-contrast imaging. No deformable mirror control was applied to mitigate the polarization aberration effect on the starlight suppression. The final impact on HabEx $\mathbf{V V}_{6}$ coronagraph performance after active wavefront control of polarization aberrations remains to be simulated and assessed during the next phase of this study.

Future work will also include modeling the other bands of HabEx's "blue channel" (550-672 nm) and HabEx's "red channel" $(672-1000 \mathrm{~nm})^{19}$ to study the effects of polarization across different wavebands. Deformable mirrors will be modeled to simulate basic adaptive optics corrections to the electric field incident on the coronagraph mask plane. Tolerance analysis of the primary mirror anisotropic coating and exoplanet simulation will reveal the allowable amount of anisotropy on the primary before the contrast becomes too limited to detect exoplanets. Additionally, investigations into other topological charges for the vector vortex coronagraph as well as other 
classes of coronagraphs will be simulated to see their effect on contrast when polarization aberrations are included. Unlike the diagonal elements, the leakage terms (i.e. XY and YX) are not maximum at the center of the coronagraph image plane which leads to a degradation in the starlight suppression of vector vortex coronagraphs. Odd-order vector vortex coronagraphs will be examined analytically as a mitigation strategy.

\section{ACKNOWLEDGEMENTS}

This work was supported by the NASA Science Mission Directorate (SMD) Astrophysics Division, technology development for exoplanet missions (TDEM) research grant No. NNX17AB296.

\section{REFERENCES}

[1] Garreth Ruane, Dimitri Mawet, Bertrand Mennesson, Jeffrey Jewell, and Stuart Shaklan. Vortex coronagraphs for the Habitable Exoplanet Imaging Mission (HabEx) concept: theoretical performance and telescope requirements. arXiv preprint arXiv:1803.03909, 2018.

[2] Wai Sze Tiffany Lam and Russell Chipman. Balancing polarization aberrations in crossed fold mirrors. Applied optics, 54(11):3236-3245, 2015.

[3] Kunjithapatham Balasubramanian, Stuart Shaklan, Amir Give'on, Eric Cady, and Luis Marchen. Deep uv to nir space telescopes and exoplanet coronagraphs: a trade study on throughput, polarization, mirror coating options and requirements. In Techniques and Instrumentation for Detection of Exoplanets $V$, volume 8151, page 81511G. International Society for Optics and Photonics, 2011.

[4] M.W. McCall, I.J. Hodgkinson, and Q. Wu. Birefringent Thin Films And Polarizing Elements (2nd Edition). World Scientific Publishing Company, 2014.

[5] JR Gee, IJ Hodgkinson, and PW Wilson. Reflection anisotropy in evaporated aluminum: Consequences for telescope mirror coatings. Journal of Vacuum Science \&3 Technology A: Vacuum, Surfaces, and Films, 4(4):1875-1878, 1986.

[6] James B Breckinridge and Ben R Oppenheimer. Polarization Effects in Reflecting Coronagraphs for WhiteLight Applications in Astronomy. The Astrophysical Journal, 600(2):1091, 2004.

[7] Garam Yun, Karlton Crabtree, and Russell A Chipman. Three-dimensional polarization ray-tracing calculus I: definition and diattenuation. Applied optics, 50(18):2855-2865, 2011.

[8] James P McGuire and Russell A Chipman. Diffraction image formation in optical systems with polarization aberrations. I: Formulation and example. JOSA A, 7(9):1614-1626, 1990. 
[9] James B Breckinridge, Wai Sze T Lam, and Russell A Chipman. Polarization Aberrations in Astronomical Telescopes: the Point Spread Function. Publications of the Astronomical Society of the Pacific, 127(951):445, 2015 .

[10] Airy Optics. http://www.airyoptics.com/. Accessed: 2018-05-16.

[11] R.A. Chipman, W.S.T Lam, and G. Young. Polarized Light and Optical Systems. CRC Press, 2018.

[12] Brian Daugherty. Demonstration of a form birefringence measurement methodology for large mirrors. in progress.

[13] Marc J Kuchner and Wesley A Traub. A Coronagraph with a Band-Limited Mask for Finding Terrestrial Planets. The Astrophysical Journal, 570(2):900, 2002.

[14] D Mawet, Pierre Riaud, Olivier Absil, and Jean Surdej. Annular groove phase mask coronagraph. The Astrophysical Journal, 633(2):1191, 2005.

[15] Grover A Swartzlander Jr. The optical vortex coronagraph. Journal of Optics A: Pure and Applied Optics, 11(9):094022, 2009.

[16] Remi Soummer, Laurent Pueyo, Anand Sivaramakrishnan, and Robert J Vanderbei. Fast computation of Lyot-style coronagraph propagation. Optics Express, 15(24):15935-15951, 2007.

[17] Rumiko Yamaguchi, Toshiaki Nose, and Susumu Sato. Liquid Crystal Polarizers with Axially Symmetrical Properties. Japanese journal of applied physics, 28(9R):1730, 1989.

[18] Scott C McEldowney, David M Shemo, and Russell A Chipman. Vortex retarders produced from photoaligned liquid crystal polymers. Optics express, 16(10):7295-7308, 2008.

[19] Stefan Martin, Mayer Rud, Paul Scowen, Daniel Stern, Joel Nissen, and John Krist. HabEx space telescope optical system. In UV/Optical/IR Space Telescopes and Instruments: Innovative Technologies and Concepts VIII, volume 10398, page 1039805. International Society for Optics and Photonics, 2017. 